\title{
Polymer brush: a promising grafting approach to scaffolds for tissue engineering
}

\author{
Woonjung Kim E Jongjin Jung * \\ Department of Chemistry, Hannam University, Daejeon 34054, Korea
}

Polymer brush is a soft material unit tethered covalently on the surface of scaffolds. It can induce functional and structural modification of a substrate's properties. Such surface coating approach has attracted special attentions in the fields of stem cell biology, tissue engineering, and regenerative medicine due to facile fabrication, usability of various polymers, extracellular matrix (ECM)-like structural features, and in vivo stability. Here, we summarized polymer brush-based grafting approaches comparing self-assembled monolayer (SAM)-based coating method, in addition to physico-chemical characterization techniques for surfaces such as wettability, stiffness/ elasticity, roughness, and chemical composition that can affect cell adhesion, differentiation, and proliferation. We also reviewed recent advancements in cell biological applications of polymer brushes by focusing on stem cell differentiation and 3D supports/implants for tissue formation. Understanding cell behaviors on polymer brushes in the scale of nanometer length can contribute to systematic understandings of cellular responses at the interface of polymers and scaffolds and their simultaneous effects on cell behaviors for promising platform designs. [BMB Reports 2016; 49(12): 655-661]

\section{INTRODUCTION}

Polymer brush is a soft material unit with an entangled structure that is covalently tethered on the surface of scaffolds or substrates $(1,2)$. Polymer brush can assign and tailor diverse structural and functional features of polymers on the scaffold or support surface. Its easy fabrication has made it applicable in various fields such as electronics, sensors, anti-fouling, catalysis, purification, and energy $(1,2)$.

There have been recent advancements in the coating process of polymer brush for stem cell biology, tissue

${ }^{*}$ Corresponding author. Tel: +82-42-629-8938; Fax: +82-42-6298811; E-mail: jongjin9@hnu.kr

https://doi.org/10.5483/BMBRep.2016.49.12.166

Received 28 September 2016

Keywords: Cell behaviors, Grafting-to/-from approach, Polymer brush, Stem cell engineering, Tissue formation engineering, and regenerative medicine $(1,3)$. This is due to the facile attachment of bioactive materials to polymer brush which can stimulate cells to control in specific biological directions, diverse scaffold materials for polymer brush coating, and simple fabrication and conjugation process. In addition, various functional polymers can be used in almost unlimited ways, and polymer chain length, density, and microstructures of polymer brush can be easily adjusted. It can not only mimic extracellular matrix (ECM)-like structures to induce cell adhesion and growth but it has been reported to be highly stable in vivo that it is considered as an optimal candidate in biomedical implants (3).

For biomedical implant to be successively developed clinically, biocompatibility of implant materials need to be outstanding. Cell adhesion and proliferation also need to be active so that it can be incorporated onto the host while having good inflammatory resistance and smooth tissue reorganization $(4,5)$. For this purpose, research is being actively conducted to constitute and control the microenvironments on scaffold surface using polymer brush to enhance cell compatible properties and modulate stem cell differentiation on its tailored surface.

This review summarizes recently reported studies on polymer brush coating for the regulation of cell behaviors for bioapplications in tissue engineering and regenerative medicine and cellular responses toward microstructures stemmed from precisely implemented nanometer-sized polymer brush. Recent research in stem cell engineering using polymer brush will be introduced along with studies based on its application in implants and three dimensional (3D) structures with the polymer brush. The effects of microstructures formed by polymer brush in such a microenvironment and materials that result in complex effects on cells will also be reviewed.

In order to effectively apply polymer brush in tissue engineering and regenerative medicine, a prior understanding is needed for the procedure of polymer brush fabrication, its physico-chemical features, and the analytic processes for characterizing them. Therefore, the properties of polymer brush and its analysis methodologies are also described by focusing on the factors that can affect cell behaviors for its application in medical implants. 


\section{POLYMER BRUSH AND ITS CHARACTERIZATION}

There are two major approaches in tailoring the physicochemical properties of an interface by attaching various molecular constituents on the scaffold/substrate: polymer brush approach and self-assembled monolayer (SAM) approach. Both processes have been intensively applied in cell biological research because these approaches not only give a new function to the surface of substrates by attaching diverse molecules, but also result in tailored interfaces similar to an ECM's microstructure that plays important role in cellular adhesion, formation, and proliferation $(4,6)$.

SAM was developed by George Whitesides group. It describes molecular assemblies, exquisitely arranged organic molecules that spontaneously form on a substrate's surface in a single layer. Due to the self-reorganizing property of these organic elements, they can be coated meticulously. For example, alkanethiols and its assortatively designed patterns via microcontact printing and dip-pen nanolithography on gold thin films of silica glass are widely used in cell research. Regardless of the advantages of SAM, limited types of useable organic molecules, unconfirmed stability on other substrates than gold, low in vivo stability, and the complex fabrication process have limited its application in cell biology and tissue engineering.

The polymer brush approach covalently tethers polymer chains on the surface of chemically reactive substrates. It has attracted special attention due to its facile fabrication process, compatibility with various scaffold materials such as glass, silicon, gold, silver, and titanium, flexibility in adjusting polymer chain length and density, and the possibility to conjugate additional functional molecules. Polymer brush approach can be divided as grafting-to and grafting-from techniques depending on the fabrication process. The grafting-to technique covalently anchors the polymer chain or polymer unit onto the reactive surface of the scaffold while the grafting-from technique forms a polymer chain or polymer unit via polymerization from polymeric initiating moiety on the scaffold surface. The grafting-to technique forms a looser brush density than the grafting-from technique because of steric hindrance caused by the previously attached polymers in its coupling process at the interface (Fig. 1A, B). According to its fabrication approaches and related parameters, polymer chain length is adjustable (2). Various attributes of polymer brush compared to SAM approach are summarized in Table 1.

For implants to be successfully integrated into the host system, cell adhesion, formation, proliferation, and differentiation have to occur actively on the scaffold-polymer brush interface. The polymer brush on a scaffold that can influence such cell behaviors needs to be quantitatively analyzed. Hydrophobicity, roughness, stiffness/elasticity, and chemical composition are important factors that can affect cell behaviors and manipulation. Generally, as the surface hydrophobicity rises, the rate of cell attachment and spreading will drop.
However, as can be seen in polyethylene glycol (PEG) and poly (sulfobetaine methacrylate) (polySBMA), the hydrophilicity of polymer brush does not always result in improved cell adhesion $(7,9,10)$. Surface hyrophobicity and hydrophilicity can be measured by the wettability via the increase and decrease in surface tension of liquid drops formed on the substrate surface. This can be quantified by measuring the static contact angle. Whereas angle measured from the surface rises in droplets on hydrophobic surfaces, it drops on hydrophilic surfaces because the droplets spread flat (11).

Roughness, a topological property of a surface, plays an important role in cell adhesion, morphogenesis, and proliferation, especially in implant and tissue formation (7). Scanning electron microscopy (SEM) is usually used to observe

A)

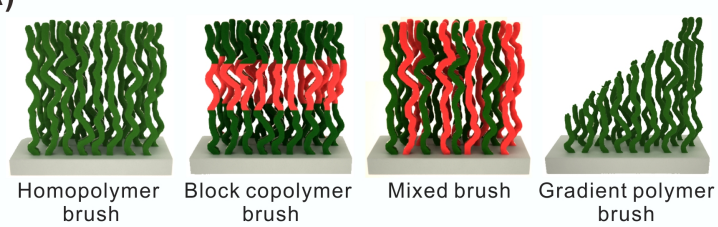

B)

Grafting-to approach

Grafting-from approach

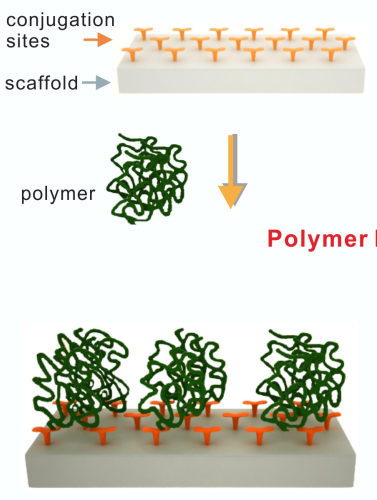

chemical conjugation between polymers and scaffold
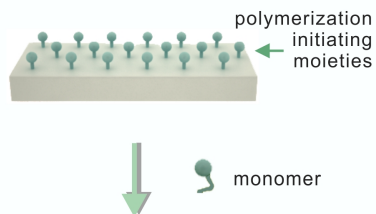

monomer

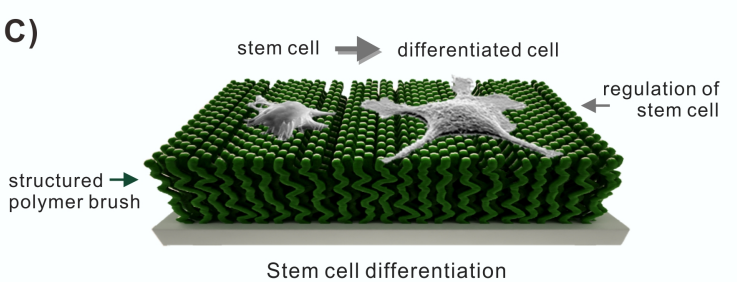

Fig. 1. (A) Overview of different types of polymer brushes (homopolymer brush, block copolymer brush, mixed brush, and gradient polymer brush). (B) Fabrication of polymer brushes on scaffolds (Grafting-to approach vs. Grafting-from approach), adapted from ref. 2. (C) Regulation of stem cell differentiation on polymer brush. 
Table 1. Comparison of the physico-chemical properties between polymer brush and self-assembled monolayer (SAM)

\begin{tabular}{|c|c|c|c|}
\hline & \multicolumn{2}{|c|}{ Polymer brush } & \multirow{2}{*}{ SAM (self-assembled monolayer) } \\
\hline & Grafting-to & Grafting-from & \\
\hline Grafting molecule & \multicolumn{2}{|c|}{ Almost all types of polymers } & Mainly alkanethiol \& alkyl silnae \\
\hline Micro-architecture & \multicolumn{2}{|c|}{ Various and complex polymeric structures } & Well assembled molecular monolayer \\
\hline Scaffold materials & \multicolumn{2}{|c|}{ Glass, titanium, gold, silver, silicon, etc } & Gold thin film, oxide-formed substrate \\
\hline Thickness & \multicolumn{2}{|c|}{ High tenability by adjusting polymer chain length } & Thin: one molecular layer \\
\hline Coating defects & \multicolumn{2}{|c|}{$\begin{array}{l}\text { Presence: short polymer chain } \\
\text { Self-healing of defects: long polymer chain }\end{array}$} & Presence of defects and pinhole \\
\hline In vivo stability & \multicolumn{2}{|l|}{ High stability } & Low stability \\
\hline Coating density & Loosely packed & Densely packed & Densely packed \\
\hline Fabrication approach & $\begin{array}{l}\text { Various chemical coupling } \\
\text { between polymer and surface }\end{array}$ & $\begin{array}{l}\text { Various polymerization } \\
\text { on the surface }\end{array}$ & Thiol-gold bond \& silane linkage \\
\hline
\end{tabular}

Table 2. Characterization of the physico-chemical properties of polymer brushes on scaffold focusing on factors that can affect cell behaviors

\begin{tabular}{|c|c|c|c|c|c|}
\hline & Wettability & Roughness & Rigidity/elasticity & Chemical composition & Height \\
\hline $\begin{array}{l}\text { Tools for } \\
\text { analysis }\end{array}$ & $\begin{array}{l}\text { Contact angle } \\
\text { measurement }\end{array}$ & AFM, SEM & AFM (force mode) & $\begin{array}{l}\text { IR \& Raman spectrometery, } \\
\text { XPS, TGA }\end{array}$ & Ellipsometry, AFM \\
\hline Parameters & $\begin{array}{l}\text { Angle between surface } \\
\text { of a liquid drop and } \\
\text { substrate }\end{array}$ & $\begin{array}{l}\text { Root mean square } \\
\text { of the height } \\
\text { of surface contour }\end{array}$ & $\begin{array}{l}\text { The degree of deflection } \\
\text { of AFM cantilever } \\
\text { at contact point }\end{array}$ & $\begin{array}{l}\text { Chemical functional group, } \\
\text { element composition, } \\
\text { thermal decomposition }\end{array}$ & $\begin{array}{l}\text { Height from the } \\
\text { surface of scaffold }\end{array}$ \\
\hline Properties & $\begin{array}{l}\text { Hydrophobicity \& } \\
\text { hydrophilicity }\end{array}$ & Topology \& geometry & Mechanical strength & $\begin{array}{l}\text { Presence of target } \\
\text { polymers on the surface }\end{array}$ & $\begin{array}{l}\text { Thickness of } \\
\text { polymer brush }\end{array}$ \\
\hline
\end{tabular}

the overall surface morphology. Atomic force microscopy (AFM) is normally used to quantitatively measure surface roughness more elaborately. Roughness via AFM is quantitatively represented by taking the root mean square of the difference between the individual peaks and the average height within specific lines or areas $(12,13)$.

Rigidity/elasticity, also referred to as stiffness/softness, of the mechanical property of a substrate is one influential parameter that can affect cell attachment, growth, and differentiation (7). Tilghman et al. have shown that a cell culture plate where the surface rigidity is modulated can cause various types of cultivated cancer cells to alter their growth, spreading, proliferation, and migration (8). Surface rigidity/elasticity can be measured in the AFM force mode instead of the image mode. While the cantilever of the AFM tip is adhered to the surface, it measures the degree of deflection depending on the surface softness/hardness of a substance.

Chemical compositions of a surface need to be analyzed to determine if polymers or biopolymers are appropriately attached to the surface. Infrared (IR) and Raman spectrometry can be used to identify the substances of the functional groups of chemicals/polymers. X-ray photoelectron spectroscopy
(XPS) can be used to analyze the electronic state and element composition of the surface. Thermal gravimetric analysis (TGA) can be utilized to detect mass reduction by a rise in temperature, ultimately monitoring the unique phase transition caused by vaporization, sublimation, and adsorption according to its own physicochemical properties.

In addition, ellipsometry and AFM can be used to measure the length of a folded polymer chain (height from bare scaffold). Cyclic voltammetry (CV) or surface zeta potential can be used to determine the electrochemical properties of a substance. Fluorescence image analysis via conjugation of fluorescent materials is obtained to identify the overall coverage of the target molecules. All these parameters and apparatuses for investigating surface properties are summarized in Table 2, focusing on factors that can influence cell behaviors.

These tools for analysis enable the micro-architecture and physico-chemical properties of a polymer brush to be characterized precisely, which can directly correlate its features with cell behaviors to aid the designing of basic coating platform using polymer brushes in tissue engineering and regenerative medicine. 


\section{POLYMER BRUSH FOR TISSUE ENGINEERING}

Polymer brush has been widely used for protein adsorption, biosensing, anti-fouling, cell culture, and regulation of cell behavior in the field of biomedicine. Cellular responses toward bioactive polymer brush and its control on cell behaviors have been especially studied in tissue engineering. Cellular manipulation using thermoresponsive polymer brush is also being actively studied.

Currently, studies on polymer brush have expanded from regulating stem cell behaviors to $3 \mathrm{D}$ support/implant coating for its application in regenerative medicine and clinical biomedicine. The delicately designed micro-architectures of polymer brush of nanometer-sized chain length and the resulting cellular responses to both polymer and scaffold materials are being systematically investigated (Fig. 1C). Such studies on polymer brush for tissue engineering will collectively contribute to the design of the most optimal platform for clinical implants.

Stem cell studies on polymer brush have been directed toward regulating stem cell behaviors by using polymer brush itself or bioactive properties of additionally attached biopolymers $(18,21)$. Poly[2(methacryloyloxy)ethyl dimethyl-(3ulfopropyl) ammonium hydroxide] (PMEDSAH) polymer brush has been shown to be able to maintain undifferentiated human embryonic stem cell (hESC) in a long-term culture (19). Poly(OEGMA-CO-HEMA) brushes conjugated with vitronectin (VN) peptide has been developed as a platform to culture human induced pluripotent stem cell (hiPSC) for a long period (17). Growth factors attached to poly (acrylic) acid (PAA) brush have been reported to be able to regulate the differentiation of mouse embryonic stem cell (mESC) (20). Thermoresponsive poly(N-isopropylacrylamide) (PNIPAAm) brush has been demonstrated to be able to control fibrinogen adhesion according to temperature for the study of the adhesion of human mesenchymal stem cell (hMSC) (21). Block copolymer (Pluronic F-127: PF127) brush conjugated with antimicrobial peptide and RGD peptide can effectively promote the antibacterial property and cell adhesion/spreading in tissue engineering (22). Beyond these studies on the effects of bioactive molecules on stem cells, recent research studies have advanced the modulation of micro-architectures of polymer brush and its properties (23, 24, 30-32). The effects of polymer brush on stem cells caused by its geometrical features are also reported (25-29).

Kumar et al. (30) have shown that reaction time and catalyst ratio of PMEDSAH in its grafting-from polymerization can control the thickness of the polymer brush via a statistical model. They have predicted and proved that variation in thickness can induce change in wettability, which subsequently change the arrangement and structure of individual polymer (30). Based on the published prediction and modification of the elaborate microstructure of polymer brush, an appropriate PMEDSAH polymer brush architecture has been proposed for the best culture condition and propagation of human embryonic stem cell (hESC) (31). The thickness of polymer brush and the resulting frictional and mechanical properties such as lateral deformation that can affect the adhesion and morphogenesis of human mesenchymal stem cells (hMSC) have been reported (32). Fabrication of diverse nanopatterns with anti-fouling polymer and cell adhesion biopolymer has been conducted to determine their effects on the spreading and differentiation of epidermal stem cells (23). In addition, the topological effects of the surface of a substrate and decoupling topological effects by polymer brush coating on hMSC have been presented (24).

Gautrot and Connelly et al. have fabricated a round-shaped geometry pattern using cell resistant poly(oligo(ethylene glycol methacrylate)) (POEGMA) polymer and ECM protein using lithography technique. They have also determined the way how geometry can affect epidermal stem cell differentiation and the formation of micro-epidermis that mimic normal epidermal tissues (25-29). These studies allow the prediction of stem cell formation influenced by microstructures and the physico-chemical properties of a polymer brush, thus contributing to the design of the platform of polymer brush for actual implants.

Most medical implants have three-dimensional structures. Research in polymer brush fabrication of 3D scaffold is actively underway to study cell adhesion and tissue formation on such 3D support. Gunnewiek et al. used PCL (ع-polycaprolactone) fiber to form 3D microporous scaffolds, which was grafted with a POEGMA brush. ECM proteins were additionally conjugated gradiently on the $3 \mathrm{D}$ substrate with the assistance of polymer brush and subsequently increased the adhesion of hMSC on the 3D support (33). It has been reported that a titanium-based bone implant coated with a POEGMA brush was successfully implanted on a rat's leg after attachment of fibronectin fragments, and it promoted the integration of tissue on this coated bone implant (34).

Hydrogel-based support is a 3D architecture/scaffold that is in the limelight because of its formation of artificial ECM network. Its fabrication methodology of bioresponsive brush by conjugating biopolymers on a hydrogel scaffold is being researched actively. It was reported that the bioactivity of a retinal precursor cell (RPC) was tested on an agarose hydrogel that patternized the protein brush of sonic hedgehog $(\mathrm{SHH})$ and ciliary neurotrophic factor (CNTF) in 3D hydrogel using a two photon irradiation method (35). Patternized RGD in poly(ethylene glycol) (PEG) - diacrylate (PEGDA) 3D hydrogel using two photon absorption lithography can affect cell confinement and migration (36). PEG-based hydrogel simultaneously coupled with integrin-mimicked peptide and matrix metalloproteinase (MMP) substrate has been fabricated to study cell invasion for tissue regeneration or bone regeneration $(37,38)$.

Besides stem cell engineering on polymer brush and polymer brush coating for 3D support, the delicate modulation 
of the thickness and density of a polymer brush is also an important factor that can affect cell behaviors due to resulting changes in its micro-architectures and physico-chemical characteristics. A fabrication process for gradient polymer brush grafting has been developed to control its chain length and density (43-45). This process can result in various degrees of protein adsorption and cell adhesion according to the gradation of polymer brushes (39). Along with polymer chain length, the microstructure of poly[\{2-(methacryloyloxy) ethyl $\}$ dimethyl-(3-sulfopropyl) ammonium hydroxide] (PMEDSAH) brush can change. Simulation results have shown that well-modulated thickness of polymer brush can affect its changes in micro-architecture and transition of physical properties such as wettability which make it possible to design the most optimal polymer brush for stem cell culture (30). A simulation of nuclear pore complex (NPC) based on polymer brush model has shown that NPC proteins placed in the different sections of the nuclear pore can form various disordered polypeptides as a conserved functional feature, and formation of the unique biopolymer brush architecture can play an important role in regulating the open-close of the nuclear pore to transport biological cargos (40).

Interestingly, as the polymer chain is shortened within a few nanometers, the microstructure and its chemical features also changed. Then cells not only interact with polymer brush, but also with scaffolds, the base materials. Gon et al. have sparsely attached about $10 \mathrm{~nm}$ cationic polymer using poly(L-lysine) (PLL)-PEG graft copolymers in anti-fouling PEG polymer brush at 7-17 nm in height. They observed that bacteria would cling to the rare cationic polymers on the bacterial-repellent PEG-brush surface by compressing the nanometer-length of PEG brush. This result leads to a deep intuition for cellular interaction at its interface on short length polymer, low density polymer, formation of flaw, and heterogeneous coating, all of which can be generated during polymer brush fabrication (41).

Chiang et al. have observed that rat basophilic leukemia (RPL) mast cells can adhere to poly(acrylic acid) (PAA) polymer brush, a cell repellent, when it is formed into square patterns smaller than RBL cells (42). In addition, cell membrane accumulates on patterned anti-fouling area (42). However, at $8 \mathrm{~nm}$ brush height, regardless of low cell membrane accumulation, there is still active cell attachment, indicating that there is a strong interaction between cells and silicon, a scaffold substance that has cell affinity (42). These results have shown that, as polymer chain length shortens, its micro-architectures and physico-chemical properties of the polymer brush will also change. In addition, it can cause simultaneous interaction between cell and scaffold and between cell and polymer. All of them need to be considered important factors when applying polymer brush in tissue engineering and regenerative medicine.

\section{CONCLUSIONS AND PERSPECTIVES}

Polymer brush is the most optimal scaffold surface grafting approach for biomedical implant, regenerative medicine, and tissue engineering. It is supported by current intensive researches on the use of polymer brush in stem cell engineering and polymer brush coating for 3D support in tissue engineering. The types of polymer used in polymer brush, polymer chain length, and brush density can modulate the microstructures and physico-chemical properties of polymer brush. It can lead to derive the most optimal cultivation condition for stem cell engineering. Furthermore, shortening polymer chain length by a few nanometers to induce simultaneous interaction between polymer and scaffold materials with cells can be used to predict the effects of low density brush, heterogeneous coating, and flaw during grafting on cellular response and tissue formation. As a result, the planning and fabrication of various micro-architecture of polymer brush, precise analysis and modulation of its physico-chemical properties, and systematic studies on its cellular responses will contribute to the optimal design of polymer brush for biomedical implant application.

\section{ACKNOWLEDGEMENTS}

We thank Mr. Gyoung Chan Seo for helping us with the figure. We also thank Dr. Soosang Chae for his valuable suggestions for the manuscript. This work (C0395024) was supported by Business for Cooperative R\&D between Industry, Academy, and Research Institute funded Korea Small and Medium Business Administration in 2016. It was also supported by Hannam University Research Fund.

\section{REFERENCES}

1. Krishnamoorthy $M$, Hakobyan $S$, Ramstedt $M$ and Gautrot JE (2014) Surface-initiated polymer brushes in the biomedical field: applications in membrane science, biosensing cell culture regenerative medicine and antibacterial coatings. Chem Rev 114, 10976-11026

2. Azzaroni O (2012) Polymer brushes here, there, and everywhere: Recent advances in their practical applications and emerging opportunities in multiple research fields. J Polym Sci A Polym Chem 50, 3225-3258

3. Moroni L, Gunnewiek MK and Benetti EM (2014) Polymer brush coatings regulating cell behavior: Passive interfaces turn into active. Acta Biomater 10, 2367-2378

4. Raynor JE, Capadona JR, Collard DM, Petrie TA and Garcia AJ (2009) Polymer brushes and self-assembled monolayers: versatile platforms to control cell adhesion to biomaterials (Review). Biointerphases 4, FA3-FA16

5. Von der Mark K, Park J, Bauer S and Schmuki P (2010) Nanoscale engineering of biomimetic surfaces: cues from the extracellular matrix. Cell Tissue Res 339, 131-153

6. Love JC, Estroff LA, Kriebel JK, Nuzzo RG and Whitesides GM (2005) Self-assembled monolayers of thiolates on 
metals as a form of nanotechnology. Chem Rev 105, 1103-1170

7. Chang HI and Wang Y (2011) Cell responses to surface and architecture of tissue engineering scaffolds, Regenerative medicine and tissue engineering - cells and biomaterials. www.intechopen.com.

8. Tilghman RW, Cowan CR, Mih JD et al (2010) Matrix rigidity regulates cancer cell growth and cellular phenotype. PLoS One 5, e12905

9. Jokerst JV, Lobovkina T, Zare RN and Gambhir SS (2011) Nanoparticle PEGylation for imaging and therapy. Nanomedicine 6, 715-728

10. Chang Y, Higuchi A, Shih YJ et al (2012) Bioadhesive control of plasma proteins and blood cells from umbilical cord blood onto the interface grafted with zwitterionic polymer brushes. Langmuir 28, 4309-4317

11. Vogler EA (1999) Water and the acute biological response to surfaces. J Biomater Sci Polym Ed 10, 1015-1045

12. Washburn NR, Yamada KM, Simon Jr CG, Kennedy SB and Amis EJ (2004) High-throughput investigation of osteoblast response to polymer crystallinity: influence of nanometer-scale roughness on proliferation. Biomaterials $25,1215-1224$

13. Vagaská B, Bacakova L, Filova E and Balik K (2010) Osteogenic cells on bio-inspired materials for bone tissue engineering. Physiol Res 59, 309-322

14. Webster KD, Crow A and Fletcher DA (2011) An AFM-based stiffness clamp for dynamic control of rigidity. PLoS One 6, e17807

15. Engler AJ, Sen S, Sweeney HL and Discher DE (2006) Matrix elasticity directs stem cell lineage specification. Cell 126, 677-689

16. Manifacier JC, Gasiot J and Fillard JP (1976) A simple method for the determination of the optical constants $n, k$ and the thickness of a weakly absorbing thin film. J Phys E: Sci Instrum 9, 1002-1004

17. Deng Y, Zhang X, Zhao X et al (2013) Long-term self-renewal of human pluripotent stem cells on peptide-decorated poly (OEGMA-Co-HEMA) brushes under fully defined conditions. Acta Biomater 9, 8840-8850

18. Tsai HA, Shen CN and Chang YC (2012) Use of surface properties to control the growth and differentiation of mouse fetal liver stem/progenitor cell colonies. Biomacromolecules 13, 3483-3493

19. Villa-Diaz LG, Nandivada H and Ding J (2010) Synthetic polymer coatings for long-term growth of human embryonic stem cells. Nat Biotechnol 28, 581-583

20. Psarra E, Foster E and König U (2015) Growth Factor-Bearing Polymer Brushes-Versatile Bioactive Substrates Influencing Cell Response. Biomacromolecules 16, 3530-3542

21. Psarra E, König U, Ueda $Y$ et al (2015) Nanostructured biointerfaces: nanoarchitectonics of thermoresponsive polymer brushes impact protein adsorption and cell adhesion. ACS Appl Mater Interfaces 7, 12516-12529

22. Muszanska AK, Rochford ET, Gruszka A et al (2014) Antiadhesive polymer brush coating functionalized with antimicrobial and RGD peptides to reduce biofilm formation and enhance tissue integration.
Biomacromolecules 15, 2019-2026

23. Gautrot JE, Malmström J, Sundh M, Margadant C, Sonnenberg A, Sutherland DS (2014) The nanoscale geometrical maturation of focal adhesions controls stem cell differentiation and mechanotransduction. Nano Lett 14, 3945-3952

24. Gunnewiek MK, Benetti EM, Luca AD, Blitterswijk CA, Moroni L and Vancso GJ (2013) Thin polymer brush decouples biomaterial's micro-/nanotopology and stem cell adhesion. Langmuir 29, 13843-13852

25. Gautrot JE, Wang C, Liu X et al (2012) Mimicking normal tissue architecture and perturbation in cancer with engineered micro-epidermis. Biomaterials 33, 5221-5229

26. Connelly JT, Mishra A, Gautrot JE and Watt FM (2011) Shape-induced terminal differentiation of human epidermal stem cells requires p38 and is regulated by histone acetylation. PLoS One 6, e27259

27. Connelly JT, Gautrot JE, Trappmann B et al (2010) Actin and serum response factor transduce physical cues from the microenvironment to regulate epidermal stem cell fate decisions. Nat Cell Biol 12, 711-718

28. Gautrot JE, Trappmann B, Connelly J et al (2010) Exploiting the superior protein resistance of polymer brushes to control single cell adhesion and polarisation at the micron scale. Biomaterials 31, 5030-5041

29. Tan KY, Lin H, Ramstedt M, Watt FM, Huck WT and Gautrot JE (2013) Decoupling geometrical and chemical cues directing epidermal stem cell fate on polymer brush-based cell micro-patterns. Integr Biol 5, 899-910

30. Kumar R and Lahann J (2016) Predictive Model for the Design of Zwitterionic Polymer Brushes: A Statistical Design of Experiments Approach. ACS Appl Mater Interfaces 8, 16595-16603

31. Qian X, Villa-Diaz LG, Kumar R, Lahann J and Krebsbach $\mathrm{PH}$ (2014) Enhancement of the propagation of human embryonic stem cells by modifications in the gel architecture of PMEDSAH polymer coatings. Biomaterials $35,9581-9590$

32. Gunnewiek MK, Ramakrishna SN, Luca AD, Vancso GJ, Moroni L and Benetti EM (2016) Stem-Cell Clinging by a Thread: AFM Measure of Polymer-Brush Lateral Deformation. Adv Mater Interfaces 3, 1500456-1500468

33. Gunnewiek MK, Luca AD, Bollemaat HZ et al (2015) Creeping Proteins in Microporous Structures: Polymer Brush Assisted Fabrication of 3D Gradients for Tissue Engineering. Adv Healthc Mater 4, 1169-1174

34. Petrie TA, Raynor JE, Dumbauld DW et al (2010) Multivalent integrin-specific ligands enhance tissue healing and biomaterial integration. Sci Transl Med 2, $45 \mathrm{ra} 60$

35. Wylie RG, Ahsan S, Aizawa Y, Maxwell KL, Morshead CM and Shoichet MS (2011) Spatially controlled simultaneous patterning of multiple growth factors in three-dimensional hydrogels. Nat Mater 10, 799-806

36. Hahn MS, Miller JS and West JL (2006) Three dimensional biochemical and biomechanical patterning of hydrogels for guiding cell behavior. Adv Mater 18, 2679-2684

37. Lutolf MP, Lauer-Fields JL, Schmoekel HG et al (2003) Synthetic matrix metalloproteinase-sensitive hydrogels for the conduction of tissue regeneration: engineering 
cell-invasion characteristics. Proc Natl Acad Sci U S A $100,5413-5418$

38. Lutolf MP, Weber FE, Schmoekel HG et al (2003) Repair of bone defects using synthetic mimetics of collagenous extracellular matrices. Nat Biotechnol 21, 513-518

39. Li L, Nakaji-Hirabayashi T, Kitano H, Ohno K, Kishioka T and Usui Y (2016) Gradation of proteins and cells attached to the surface of bio-inert zwitterionic polymer brush. Colloids and Surfaces B: Biointerfaces 144, 180-187

40. Ando D, Zandi R, Kim YW, Colvin M, Rexach $M$ and Gopinathan A (2014) Nuclear pore complex protein sequences determine overall copolymer brush structure and function. Biophys J 106, 1997-2007

41. Gon S, Kumar KN, Nusslein K and Santore MM (2012) How bacteria adhere to brushy peg surfaces: clinging to flaws and compressing the brush. Macromolecules 45, 8373-8381

42. Chiang EN, Dong R, Ober CK and Baird BA (2011) Cellular responses to patterned poly (acrylic acid) brushes. Langmuir 27, 7016-7023

43. Gosecka M, Pietrasik J, Decorse P et al (2015) Gradient Poly (styrene-co-polyglycidol) Grafts via Silicon Surface-Initiated AGET ATRP. Langmuir 31, 4853-4861

44. Gallant ND, Lavery KA, Amis EJ and Becker ML (2007) Universal gradient substrates for "click" biofunctionalization. Adv Mater 19, 965-969

45. Shida N, Koizumi $\mathrm{Y}$, Nishiyama $\mathrm{H}$, Tomita I and Inagi S (2015) Electrochemically mediated atom transfer radical polymerization from a substrate surface manipulated by bipolar electrolysis: Fabrication of gradient and patterned polymer brushes. Angew Chem Int Ed 54, 3922-3926 